\title{
Experimental and Theoretical Study of Zircon and Scheelite Phases of $\mathrm{DyVO}_{4}$
}

\author{
O. Ermakova ${ }^{a, b}$, W. Paszkowicz ${ }^{a, *}$, J. López-Solano $^{c}, \mathrm{~A} \mathrm{Muñoz}^{c}$ And H. Dabkowska $^{d}$ \\ ${ }^{a}$ Polish Academy of Sciences, Institute of Physics, al. Lotników 32/46, Warszawa 02-668, Poland \\ ${ }^{b}$ Russian Academy of Sciences, Ural Division, Institute of Solid State Chemistry (ISSC), Pervomaiyskaya 91,
} Ekaterinburg 620219, Russian Federation

${ }^{c}$ Departamento de Física Fundamental II, MALTA Consolider Team and Instituto de Materiales y Nanotecnología, Universidad de La Laguna 38205, Tenerife, Spain

${ }^{d}$ Department of Physics, McMaster University, Hamilton L8S 4M1, Canada

\begin{abstract}
Polycrystalline zircon-type dysprosium orthovanadate, $\mathrm{DyVO}_{4}$, prepared from a single crystal grown by slow cooling from $\mathrm{PbO} / \mathrm{PbF}_{2}$ flux, was studied by X-ray diffraction. Rietveld refinement provided the following unit cell size and oxygen atom coordinates: $a=7.14811(4) \AA ; c=6.30825(4) \AA, V=322.323(3) \AA 3, y\left(\mathrm{O}^{2-}\right)=0.4300(4)$; $z\left(\mathrm{O}^{2-}\right)=0.2082(4)$ which are of a particularly high accuracy and show consistency with earlier reported values. Density functional theory calculations within the generalized gradient approximation for the exchange-correlation energy were also performed, providing values of structure parameters which differ by less than $2 \%$ from the experimental ones. The agreement between theory and experiment demonstrates the value of these calculations for understanding the structure of compounds of $\mathrm{RVO}_{4}$ family. In addition, density functional theory calculations were performed for the scheelite-type $\mathrm{DyVO}_{4}$; also for this polymorph the discrepancy with the only known set of lattice parameters is less than $2 \%$. Values of oxygen atom coordinates have not been reported yet for this polymorph; here, the calculated ones are quoted.
\end{abstract}

PACS: $42.70 . \mathrm{Hj} ; 61.43 . \mathrm{Bn}, 61.66 . \mathrm{Fn}$

\section{Introduction}

Materials from the family of rare-earth orthovanadates $\left(\mathrm{RVO}_{4}\right)$, mineral name wakefieldite $(\mathrm{La}, \mathrm{Ce}, \mathrm{Nd}, \mathrm{Y}) \mathrm{VO}_{4}$, exhibit unique physicochemical and optical properties [14]. These properties lead to various possible applications such as components of catalysts [5], lasers [6-9], Raman lasers [10], low-loss optical planar waveguides [11], other optical devices and phosphors [12] etc. Tsipis et al. [13] have noted that rare-earth orthovanadates are interesting for high-temperature electrochemical applications as they offer sufficient stability under the operation conditions. For $\mathrm{DyVO}_{4}$, a particularly interesting application can be found in the field of photocatalysts [14].

At ambient pressure and temperature, most of the $\mathrm{RVO}_{4}(\mathrm{R}=\mathrm{Y}, \mathrm{Ce}-\mathrm{Lu})$ compounds adopt the zircon-type structure (space group $I 4_{1} / a m d$ ). A study performed for an example of $\mathrm{GdVO}_{4}$ [15] shows that this phase may be nonstoichiometric, what leads to small but detectable lattice parameter changes of the order of $0.001 \AA$. The $\mathrm{RVO}_{4}$ compounds are known to undergo a phase transition to a scheelite-type polymorph (space group $I_{1} / a$ ) at pressures below $10 \mathrm{GPa}[16,17]$ which remains metastable after releasing the pressure. For some of them, in particular for $\mathrm{DyVO}_{4}$, a mechanochemical way for the scheelite-type phase preparation has been documented [18]. The literature data on the structure of $\mathrm{DyVO}_{4}$ are scarce: representative ones are included in Table I quoting the data from

\footnotetext{
* corresponding author; e-mail: paszk@ifpan.edu.pl
}

Refs. [4, 19-27] for zircon-type phase; for scheelite-type phase only a single data set (Ref. [28]) of low-accuracy unit-cell parameters could be found in the literature (see Table II). The reported data involve materials prepared by various methods, two of them have been made on single crystals grown by slow cooling from flux (one of them has been powdered). As for the structure refinement, only three datasets have been found, with mostly single crystal structure refinement used. The aim of the present investigation was to experimentally determine the structure of zircon-type dysprosium orthovanadate through Rietveld refinement of the X-ray diffraction data and to compare this structure with the results of density functional theory (DFT) calculations for this material.

\section{Experimental}

A DyVO $\mathrm{D}_{4}$ single crystal was grown by slow cooling method from $\mathrm{PbO} / \mathrm{PbF}_{2}$ flux, following the recipe for other rare-earth orthovanadates [29]. For powder diffraction experiments, this monocrystal was ground in an agate mortar. The resulting fine powder was studied using a laboratory Bragg-Brentano powder diffractometer (Philips X'Pert Pro MPD Alpha1, supplied by Philips/Panalytical) equipped with an incident beam Ge(111) Johannson monochromator and a strip detector. $\mathrm{Cu} \mathrm{K} \alpha_{1}$ radiation was used in the diffraction experiment. The obtained X-ray diffraction pattern served for the structure refinement of the sample. 
TABLE I

Structural data for zircon-type $\mathrm{DyVO}_{4}$. Lattice parameters $a$ and $c$, unit cell volume $V$, and oxygen atom coordinates $x$ and $y$ are provided.

\begin{tabular}{|c|c|c|c|c|c|c|c|c|c|c|c|c|}
\hline set no. & $a[\AA]$ & $c[\AA]$ & $c / a$ & $V\left[\AA^{3}\right]$ & $x\left(\mathrm{O}^{2}\right)$ & $y\left(\mathrm{O}^{2}\right)$ & $T[\mathrm{~K}]$ & H1 & $\mathrm{H} 2$ & H3 & Ref. & Year \\
\hline $1(\mathrm{C})$ & $7.1434(4)$ & $6.313(1)$ & $0.8838(2)$ & $322.14(9)$ & n.r. & n.r. & 298 & $\begin{array}{l}\text { chemical } \\
\text { reaction+drying }\end{array}$ & XRPD & LSQ & [19] & 1966 \\
\hline $2(\mathrm{C})$ & 7.135 & 6.288 & 0.8813 & 320.11 & 0.321 & 0.185 & 293 & powdered SCF & NPD & LSQ & [20] & 1971 \\
\hline $3(\mathrm{C})$ & $7.136(2)$ & $6.307(2)$ & $0.8838(5)$ & $321.2(3)$ & n.r. & n.r. & 300 & n.r. & NPD & n.r. & [21] & 1972 \\
\hline $4(\mathrm{~A})$ & $7.1483(4)$ & $6.3075(3)$ & $0.8824(1)$ & $322.30(5)$ & n.r. & n.r. & 303 & n.r. & XRPD & LSQ & [22] & 1980 \\
\hline $5(\mathrm{~A})$ & $7.1480(2)$ & $6.3067(3)$ & $0.88230(7)$ & $322.23(3)$ & n.r. & n.r. & 298 & solid state reaction & XRPD & LSQ & [23] & 1984 \\
\hline $6(\mathrm{~B})$ & $7.1429(8)$ & $6.300(2)$ & $0.8820(4)$ & $321.4(2)$ & $0.4340(7)$ & $0.2016(6)$ & 292 & $\mathrm{SCF}$ & XRSCD & SCR & [25] & 1996 \\
\hline $7(\mathrm{C})$ & 7.140 & 6.311 & 0.8839 & 321.73 & n.r. & n.r. & RT & $\begin{array}{l}\text { nanocrystalline } \\
\text { rods }\end{array}$ & XRPD & n.r. & [26] & 2004 \\
\hline $8(\mathrm{~B})$ & $7.1324(2)$ & $6.2972(3)$ & $0.8829(7)$ & $320.35(3)$ & $0.0521(2)$ & $0.2054(2)$ & RT & $\begin{array}{l}\text { hydrothermal } \\
\text { synthesis }\end{array}$ & $\mathrm{XRSCD}$ & $\mathrm{SCR}$ & {$[24]$} & 2005 \\
\hline $9(\mathrm{C})$ & 7.142 & 6.30 & 0.8821 & 321.35 & n.r. & n.r. & RT & $\begin{array}{l}\text { nanocrystalline, } \\
\text { chemical reaction }\end{array}$ & XRPD & n.r. & [27] & 2007 \\
\hline $10(\mathrm{C})$ & 7.142 & 6.308 & 0.8832 & 321.76 & n.r. & n.r. & $\mathrm{RT}$ & nanocrystalline & XRPD & n.r. & [4] & 2009 \\
\hline $11(\mathrm{~A})$ & $7.14811(4)$ & $6.30825(4)$ & $0.88251(1)$ & $322.323(3)$ & $0.4300(4)$ & $0.2082(4)$ & $300(3)$ & powdered SCF & XRPD & $\mathrm{RR}$ & this work & 2012 \\
\hline 12 & 7.2035 & 6.3233 & 0.87 & 328.1 & 0.4344 & 0.2002 & 0 & - & DFT & PBE & this work & 2012 \\
\hline 13 & 7.1174 & 6.2466 & 0.87 & 316.4 & 0.4351 & 0.1990 & 0 & - & DFT & PBEsol & this work & 2012 \\
\hline
\end{tabular}

Abbreviations: H1 - sample form or preparation, H2 - measurement/calculation, H3 - unit cell/structure refinement method or calculation model, XRPD - X-ray powder diffraction, XRSCD - X-ray single crystal diffraction, NPD — neutron powder diffraction, LSQ - least squares refinement, RR - Rietveld refinement, SCR - singlecrystal refinement, PBE - DFT calculations performed within the PBE XC functional, PBEsol — DFT calculations performed within the PBEsol XC functional, SCF — a single crystal grown from flux, n.r. - not reported. For (A), (B), (C) - see text.

TABLE II

Structural data for scheelite-type $\mathrm{DyVO}_{4}$. Lattice parameters $a$ and $c$, unit cell volume $V$, and oxygen atom coordinates $x, y$ and $z$ are provided.

\begin{tabular}{c|c|c|c|c|c|c|c|c|c|c|c|c}
\hline \hline$a[\AA]$ & $c[\AA]$ & $c / a$ & $V\left[\AA^{3}\right]$ & $x\left(\mathrm{O}^{2}\right)$ & $y\left(\mathrm{O}^{2}\right)$ & $z\left(\mathrm{O}^{2}\right)$ & $T[\mathrm{~K}]$ & $\mathrm{H} 1$ & $\mathrm{H} 2$ & $\mathrm{H} 3$ & Ref. & Year \\
\hline $5.08(2)$ & $11.24(2)$ & $2.21(1)$ & $290(2)$ & n.r. & n.r. & n.r. & RT & powder & XRPDG & n.r. & {$[28]$} & 1989 \\
5.0725 & 11.3078 & 2.2292 & 290.95 & 0.2551 & 0.1051 & 0.0446 & 0 & - & DFT & PBE & this work & 2012 \\
5.0126 & 11.1252 & 2.2184 & 279.53 & 0.2553 & 0.1033 & 0.0435 & 0 & - & DFT & PBEsol & this work & 2012 \\
\hline
\end{tabular}

XPRDG - X-ray powder diffraction using a Gandolfi camera, for other abbreviations see Table I.

\section{Density functional theory calculations}

Density functional theory calculations were performed with the VASP ab initio code [30]. Projector augmentedwave pseudopotentials $[31,32]$ with valence configurations $5 p^{6} 4 f^{1} 6 s^{2}$ for Dy, $3 p^{6} 3 d^{3} 4 s^{2}$ for $\mathrm{V}$, and $2 s^{2} 2 p^{6}$ for $\mathrm{O}$ were used. Calculations within the Perdew-BurkeErnzerhof (PBE) [33] and the more recent PBEsol [34] generalized gradient approximations of the exchangecorrelation (XC) energy functional were performed. An energy cutoff of $520 \mathrm{eV}$ and dense Monkhorst-Pack [35] grids appropriate to the zircon-type and scheelite-type structures ensured a total energy convergence of $1 \mathrm{meV}$ per formula unit. Full optimization of the lattice parameters and atomic positions at constant volume was performed, resulting in a diagonal hydrostatic stress tensor with differences of less than $0.1 \mathrm{GPa}$ between its components and atomic forces smaller than $0.005 \mathrm{eV} / \AA$. Zero point motion, temperature and magnetic effects were not included in this work.

\section{Results}

Phase analysis performed on the basis of powder Xray diffraction data ("set I") showed that the $\mathrm{DyVO}_{4}$ crystal is a single phase of zircon-type. The data were treated by the Rietveld procedure, with 19 parameters (2 lattice parameters, 2 oxygen position parameters, 3 isotropic atomic displacement parameters, 5 profile shape and width parameters, 1 preferred orientation parameter, 1 scale factor, 1 systematic error parameter, 4 peakasymmetry parameters), assuming valencies of $+3,+5$ and -2 for dysprosium, vanadium and oxygen, respectively. The refined profile is displayed in Fig. 1. To check the preferred orientation effect, various orientation directions were tried using the exponential model implemented in the Fullprof software. The effect of the preferred orientation is found to be minor, the best direction was [100], in agreement with the $\{100\}$ cleavage plane of other compounds of the same family, such as $\mathrm{YVO}_{4}$ and $\left.\mathrm{LuVO}_{4}[36,37]\right)$. The refinement results show that the studied $\mathrm{DyVO}_{4}$ crystal has the lattice parameters 
$a=7.14811(4) \AA ; c=6.30825(4) \AA$ and unit cell volume $V=322.323(3) \AA^{3}$, with the following atomic positions: $\mathrm{Dy}^{3+}$ at $(0 ; 0.75 ; 0.125), \mathrm{V}^{5+}$ at $(0 ; 0.25 ; 0.375)$, $\mathrm{O}^{2-}$ at $(0 ; 0.4300(4) ; 0.2082(4))$. Refined isotropic temperature factors for $\mathrm{Dy}^{3+}, \mathrm{V}^{5+}$ and $\mathrm{O}^{2-}$ are equal to
$0.74(1), 0.42(3)$ and $0.90(7)$, respectively; they partially differ from the respective values $(0.1,0.49,0.58)$ determined by neutron diffraction in Ref. [38]. The density derived from the unit cell is $5.71725(5) \mathrm{g} / \mathrm{cm}^{3}$.

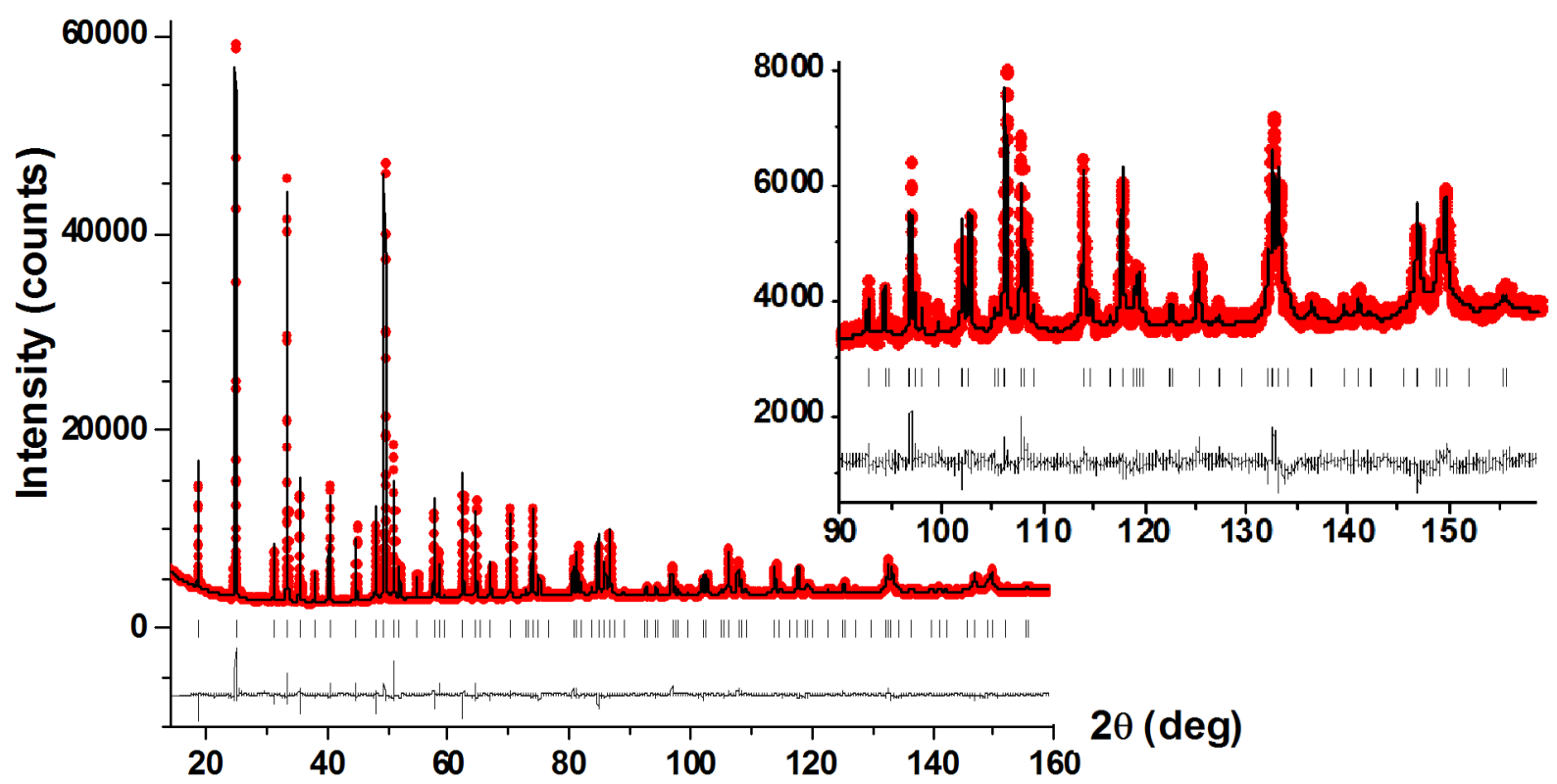

Fig. 1. Rietveld refinement for zircon-type $\mathrm{DyVO}_{4}$. Crosses represent the experimental points, the solid line stays for the fitted pattern, the difference pattern is shown in the lower part of the figure. Lower vertical bars represent the Bragg reflection positions.

It is worth noting, that our result derived from the laboratory data is, as far as we know, the first published structure refinement for zircon type $\mathrm{DyVO}_{4}$, based on powder X-ray diffraction data. Very small values of the standard deviations of the presently determined lattice parameters $\left(\ll 1 \times 10^{-4} \AA\right)$ reflect both, the high quality of the crystal studied and the very good precision of the experimental setup used. This could be achieved due to the excellent statistics and resolution connected with the use of the strip detector, a device proposed for application in Bragg-Brentano diffractometers and tested by Zięba and coworkers [39, 40] and, starting from year 2000, developed commercially by various suppliers. For this polymorph, ten datasets from earlier studies are cited in Table I. Generally, the discrepancies between the absolute values of lattice parameters are relatively large, within $\pm 0.01 \AA$, i.e. they are quite big as compared with standard deviations. Various factors may contribute to these discrepancies, such as the preparation method, nature of the sample (single crystal, polycrystal, nanocrystal), purity, kind of experiment (X-ray single diffraction, powder X-ray diffraction, neutron diffraction), etc. It will be useful to distinguish three data groups: the powder diffraction data with reported small lattice-parameter standard deviations (group I with standard-deviation values below $5 \times 10^{-4} \AA$, denoted by "A" in Table I), single crystal diffraction data (denoted as "B") and remaining ones ("C"). The observation that the data of group A differ within the error margin demonstrates that carefully conducted experiments lead to consistent unit cell sizes, irrespectively the Rietveld refinement or least squares refinement is used. The data from set B show lower absolute values, despite the good sigmas, whereas data of group $\mathrm{C}$ display a larger scatter of values. It is thus reasonable to state that the lattice parameters within the group A are the most reliable ones for zircon type $\mathrm{DyVO}_{4}$. A similar conclusion has been recently presented for the particularly difficult case of $\mathrm{CaMnO}_{3}$ compound, where such analysis could be done on the basis of still larger number of literature datasets [41].

As described in Sect. 3, the density functional theory $a b$ initio calculations were conducted using both the $\mathrm{PBE}$ and PBEsol XC functionals. For the zircon-type structure at zero pressure, the PBE calculations provide $a=7.2035 \AA, c=6.3233 \AA, V=328.119 \AA^{3}$, $y\left(\mathrm{O}^{2-}\right)=0.4344, z\left(\mathrm{O}^{2-}\right)=0.2002$, and the PBEsol ones $a=7.1174 \AA, c=6.2466 \AA, V=316.436 \AA^{3}$, $y\left(\mathrm{O}^{2-}\right)=0.4351, z\left(\mathrm{O}^{2-}\right)=0.1990$. The difference between the calculated and measured lattice parameters is below 1\%, the PBE values being overestimated and the PBEsol underestimated, following the trend already described for other bulk solids [42]. It is worth noting that 
the dimensionless quantities connected with the structure, namely the axial ratio and the oxygen atom coordinates, are quite accurately calculated using both $\mathrm{XC}$ functionals and that the difference between the oxygen position parameters obtained by the two computational approaches is negligible ( $c f$. Table I).

The scheelite-type structure has also been considered in the present DFT study. The following structure parameters were obtained for zero pressure: $a=5.0725 \AA$, $c=11.3078 \AA, V=290.953 \AA^{3}, x\left(\mathrm{O}^{2-}\right)=0.2551$, $y\left(\mathrm{O}^{2-}\right)=0.1051, z\left(\mathrm{O}^{2-}\right)=0.0446$ for the PBE XC functional, and $a=5.0126 \AA, c=11.1252 \AA, V=279.534 \AA^{3}$, $x\left(\mathrm{O}^{2-}\right)=0.2553, y\left(\mathrm{O}^{2-}\right)=0.1033, z\left(\mathrm{O}^{2-}\right)=0.0435$ for the PBEsol. A comparison of calculated lattice parameters with a low-precision experimental dataset from Ref. [28] (cf. Table II) shows that theory and experiment agree within $2 \%$. Also for this phase the difference between the oxygen position parameters calculated using the PBE and PBEsol XC functionals is marginal. It should be noted that in the PBE and PBEsol calculations the total energy of the scheelite-type phase is respectively 270 and $136 \mathrm{meV}$ per formula unit above that of the zircon-type, thus confirming the latter as the stable ambient pressure phase from the theoretical point of view.

\section{Summary}

In the present study, the structure is determined both experimentally and theoretically for zircon-type $\mathrm{DyVO}_{4}$, and theoretically for the metastable scheelitetype $\mathrm{DyVO}_{4}$. The results achieved using X-ray diffraction and $a b$ initio DFT calculations, demonstrate a good agreement between the measured and calculated parameters of both crystal structures. In the experimental part, Rietveld refinement provided the crystal structure parameters for zircon-type $\mathrm{DyVO}_{4}$, in particular the lattice parameter values $a=7.14811(4) \AA, c=6.30825(4) \AA$. These values have a markedly higher accuracy than those determined in previous studies, thus being, to the best of our knowledge, the first refinement of the crystal structure by the Rietveld method for this rare material. An excellent consistency of the present experimental data with specific earlier data is attributed to the choice of favourable diffraction-experiment conditions.

Despite the approximations used in the present DFT study, there is a good overall agreement between $a b$ initio and experimental data for both zircon-type and scheelitetype $\mathrm{DyVO}_{4}$ polymorphs, with discrepancies that do not exceed $2 \%$. For the scheelite-type phase, theoretical information on the experimentally unknown oxygen atom position is presented here for the first time, the PBE and PBEsol calculations providing fairly similar values: $x\left(\mathrm{O}^{2-}\right)=0.2551, y\left(\mathrm{O}^{2-}\right)=0.1051, z\left(\mathrm{O}^{2-}\right)=0.0446$ and $x\left(\mathrm{O}^{2-}\right)=0.2553, y\left(\mathrm{O}^{2-}\right)=0.1033, z\left(\mathrm{O}^{2-}\right)=$ 0.0435 , respectively.

\section{Acknowledgements}

The authors are grateful to Dr. Roman Minikayev (Institute of Physics, PAS) for technical help and fruitful discussions. Two authors (J.L.S. and A.M.) acknowledge the financial support of the Spanish Ministerio de Economía y Competitividad under Grants No. MAT2010-21270C04-03 and No. CSD2007-00045, and the access to the computational resources of the MALTA Cluster Computer (Universidad de Oviedo) and the Red Española de Supercomputación. The measurements performed at Hasylab have received funding from the 7 th Framework Programme (FP7/2007-2013) of European Community under ELISA grant agreement No. 226716.

\section{References}

[1] V.A. Gubanov, D.E. Ellis, A.A. Fotiev, J. Solid State Chem. 21, 303 (1977).

[2] A. Huignard, T. Gacoin, J.-P. Boilot, Chem. Mater. 12, 1090 (2000).

[3] D.E. Zelmon, J.M. Northridge, J.J. Lee, K.M. Currin, D. Perlov, Appl. Optics 49, 4973 (2010).

[4] C. Yu, M. Yu, C. Li, C. Zhang, P. Yang, J. Lin, Cryst. Growth Des. 9, 783 (2009).

[5] C.T. Au, W.D. Zhang, H.L. Wan, Catal. Lett. 37, 241 (1996).

[6] R.A. Fields, M. Birnbaum, C.L. Fincher, Appl. Phys. Lett. 51, 1885 (1987).

[7] A.I. Zagumennyi, V.G. Ostroumov, I.A. Shcherbakov, T. Jensen, J.P. Meyen, G. Huber, Sov. J. Quantum Electron. 22, 1071 (1992).

[8] P.A. Studenikin, A.I. Zagumennyi, Yu.D. Zavartsev, P.A. Popov, I.A. Shcherbakov, Quantum Electron. 25, 1162 (1995).

[9] C. Maunier, J.L. Doualan, R. Moncorgé, A. Speghini, M. Bettinelli, E. Cavalli, J. Opt. Soc. Am. B 19, 1794 (2002).

[10] A.A. Kaminskii, K. Ueda, H.J. Eichler, Y. Kuwano, H. Kouta, S.N. Bagaev, T.H. Chyba, J.C. Barnes, G.M.A. Gad, T. Murai, J Lu, Optics Commun. 194, 201 (2001).

[11] F. Chen, X. Wang, S. Li, G. Fu, K. Wang, Q. Lu, D. Shen, R. Nie, H. Ma, J. Appl. Phys. 94, 4708 (2003).

[12] M. Yu, J. Lin, S.B. Wang, Appl. Phys. A: Mater. Sci. Proc. 80, 353 (2005).

[13] E.V. Tsipis, M.V. Patrakeev, V.V. Kharton, N.P. Vyshatko, J.R. Frade, J. Mater. Chem. 12, 3738 (2002).

[14] Y. He, L. Zhao, Y. Wang, H. Lin, T. Li, X. Wu, Y. Wu, Chem. Eng. J. 169, 50 (2011).

[15] S. Osawa, T. Katsumata, T. Iyoda, Y. Enoki, S. Komuro, T. Morikawa, J. Cryst. Growth 198/199, 444 (1999).

[16] O. Fukunaga, S. Yamaoka, Phys. Chem. Miner. 5, 167 (1979).

[17] O. Muller, R. Roy, Z. Kristallogr. 138, 237 (1973).

[18] T. Tojo, Q. Zhang, F. Saito, J. Alloys Compds 427, 219 (2007).

[19] H.E. Swanson, M.C. Morris, E.H. Evans, Standard Xray Diffraction Powder Patterns, National Bureau of Standards Monograph 25, sect. 4, National Bureau of Standards, Gaithersburg 1966, p.15.

[20] G. Will, W. Schaefer, J. Phys. C 4, 811 (1971). 
[21] W. Schaefer, Berichte der Kernforschungsanlage Juelich 830, 70 (1972).

[22] N. Rajashekhar Reddy, K. Satyanarayana Murthy, K.V. Krishna Rao, J. Mater. Sci. Lett. 16, 1422 (1981).

[23] A.T. Aldred, Acta Cryst. B 40, 569 (1984).

[24] S. Mahapatra, A. Ramanan, J. Alloys Compds 395, 149 (2005).

[25] D.F. Mullica, E.L. Sappenfield, M.M. Abraham, B.C. Chakoumakos, L.A. Boatner, Inorg. Chim. Acta 248, 85 (1996).

[26] W. Fan, W. Zhao, L. You, X. Song, W. Zhang, H. Yu, S. Sun, J. Solid State Chem. 177, 4399 (2004).

[27] J. Liu, Y. Li, J. Mater. Chem. 17, 1797 (2007).

[28] S.J. Duclos, A. Jayaraman, G.P. Espinosa, A.S. Cooper, R.G. Maines Sr. J. Phys. Chem. Solids 50, 769 (1989).

[29] A. Dabkowski, H. Dabkowska, G. Jasiolek, J. LessCommon Met. 110, 255 (1985).

[30] G. Kresse, J. Hafner, J. Furthmüller, Phys. Rev. B 59, 1758 (1999).

[31] G. Kresse, D. Joubert, Phys. Rev. B 59, 1758 (1999).

[32] P.E. Blöchl, Phys. Rev. B 50, 17953 (1994).

[33] J. P. Perdew, K. Burke, M. Ernzerhof, Phys. Rev. Lett. 77, 3865 (1996).

[34] J.P. Perdew, A. Ruzsinszky, G.I. Csonka, O.A. Vydrov, G.E. Scuseria, L.A. Constantin, X. Zhou, K. Burke, Phys. Rev. Lett. 100, 136406 (2008).
[35] H.J. Monkhorst, J.D. Pack, Phys. Rev. B 13, 5188 (1976).

[36] C. Goutaudier, F.S. Ermeneux, M.T. CohenAdad, R. Moncorgé, M. Bettinelli, E. Cavalli, Mater. Res. Bull. 33, 1457 (1998).

[37] S. Zhao, H. Zhang, J. Liu, J. Wang, X. Xu, Z. Zhao, J. Xu, M. Jiang, J. Cryst. Growth 279, 146 (2005).

[38] H. Fuess, A. Kallel, J. Solid State Chem. 5, 11 (1972).

[39] A. Zięba, W. Dąbrowski, A. Czermak, in:, Proc. First Polish Meeting on High-Resolution X-ray Diffraction and Topography, Szklarska Poręba, Ed. M. Leszczyński, High Pressure Research Centre, Warsaw 1996, p. 205 (in Polish).

[40] A. Zięba, W. Białas, W. Dąbrowski, P. Gryboś, M. Idzik, B. Leśniewska, J. Kudłaty, J. Słowik, in: Applied Crystallography. Proc. of the XVIII Conference on Applied Crystallography, Wista 2000, Eds. H. Morawiec, D. Stróż, World Scientific, Singapore 2001), p. 130

[41] W. Paszkowicz, J. Pietosa, S.M. Woodley, P.A. Dluzewski, M. Kozlowski, C. Martin, Powder Diffract. 25, 46 (2011).

[42] G.I. Csonka, J.P. Perdew, A. Ruzsinsky, P.H.T. Philipsen, S. Lebègue, J. Paier, O.A. Vydrov, J.G. Ángyán, Phys. Rev. B 79, 155107 (2009). 